\title{
Massive MIMO Multicasting in Noncooperative Multicell Networks
}

\author{
Zhengzheng Xiang*, Meixia Tao* and Xiaodong Wang ${ }^{\dagger}$ \\ ${ }^{*}$ Dept. of Electronic Engineering, Shanghai Jiao Tong University, Shanghai, China \\ ${ }^{\dagger}$ Dept. of Electronic Engineering, Columbia University, New York, USA \\ Emails: \{7222838,mxtao\}@sjtu.edu.cn,wangx@ee.columbia.edu
}

\begin{abstract}
We study the massive MIMO (multiple-input multiple-output) multicast transmission in multicell networks, where each base station (BS) is equipped with a large-scale antenna array and transmits a common message using a single beamformer to multiple mobile users. We first consider the case when each BS knows the perfect channel state information (CSI) of all its served users. We show that the asymptotically optimal beamformer structure at each BS is a linear combination of the channel vectors of its multicast users. The optimal combination coefficients are also obtained in closed form. Then we consider the imperfect CSI scenario where each BS obtains the CSI through uplink channel estimation. We propose a novel pilot scheme that estimates the compound channel rather than the individual channels of multicast users in each cell. This scheme is able to completely eliminate pilot contamination. The optimal power control of pilot transmission is also derived. Numerical results show that the performance of the proposed pilot scheme with pilot power control is close to that of the perfect CSI case.
\end{abstract}

Index Terms-Physical layer multicasting, massive MIMO, asymptotic orthogonal, pilot contamination

\section{INTRODUCTION}

Wireless multicasting is a central technique of the future wireless networks. By exploiting channel state information (CSI) at the transmitter equipped with multiple antennas (also known as multiple-input multiple-output (MIMO)), physical layer multicasting in the form of beamforming is able to significantly improve the performance of wireless multicast transmissions. Unlike the traditional unicast beamforming design in multi-user or multi-cell systems, which is maturing, the core problem of multicast beamforming is generally NPhard [1] and hence more challenging. A key tool to find nearoptimal solutions is semidefinite relaxation as adopted in the existing works [1]-[3]. Other approaches such as stochastic beamforming and Alamouti space-time coding based beamforming are considered in [4]-[6].

Recently, the massive, or large-scale MIMO technology has emerged as a promising and efficient approach to deal with the severe cochannel interference and hence to address the ever-increasing demands of high mobile data traffic [7]. In [8], Marzetta first showed that by applying unlimited number of antennas at each base station (BS) of a noncooperative cellular network, the intracell interference can be substantially

This work is supported by the National 973 project under grant $2012 \mathrm{CB} 316100$ and by the NSF of China under grants 61329101 and 61221001 . reduced without sophisticated beamforming design. Moreover the transmit power at the BS can be made arbitrarily small.

While there are many advantages for the massive MIMO technique, one challenge is the acquisition of CSI at the transmitter, especially for the downlink transmission. The same issue occurs for multicast transmission. Currently, the common assumption is the time-division duplex (TDD) protocol. The BS estimates the uplink channels and obtains the downlink CSI by the channel reciprocity. However, this will suffer from the phenomenon called pilot contamination in the multicell scenario since the users in other cells may apply the same (or nonorthogonal) pilot sequences. The interference caused by pilot contamination will not vanish even when the number of BS antennas goes to infinity.

The goal of this paper is to investigate the benefits of massive MIMO on multicast transmission in cellular networks. We first consider the multicast beamforming design in the ideal case with perfect CSI. We show that the asymptotically optimal structure of the beamforming vector for each BS is a linear combination of the channel vectors of the multicast users served in each cell. Based on this structure, we further derive the optimal design parameters for each BS and obtain simple and closed-form expressions.

Then we consider the imperfect CSI case where each BS obtains the CSI through channel estimation in the uplink. To tackle the pilot contamination problem, we propose a novel pilot scheme. The key idea is to assign one pilot sequence to one cell which is shared by all the multicast users served in the cell. Moreover, the pilot sequences assigned to neighboring cells are mutually orthogonal. By doing so, the inherited pilot contamination resulting from the conventional pilot approach [8] can be avoided completely. In the meantime, each BS estimates a composite channel which is a linear combination of the individual channels of its served multicast users. Such a composite channel has the same structure as the asymptotically optimal multicast beamformer and hence can serve as the beamfomrer. In addition, by applying pilot power control among the users in each cell, we can optimize the composite channel vector and improve the performance of the network. Numerical results show that the performance of the proposed pilot scheme for composite channel estimation with pilot power control is close to that of the perfect CSI case.

Notations: Boldface uppercase letters denote matrices and boldface lowercase letters denote vectors. $\mathbb{R}, \mathbb{C}$ and $\mathbb{Z}^{+}$denote 
the sets of real numbers, complex numbers, and positive integers, respectively. $(\cdot)^{T},(\cdot)^{*},(\cdot)^{H}$ and $\operatorname{Tr}\{\cdot\}$ are the transpose, conjugate, Hermitian transpose and trace operators, respectively. $\mathbf{I}_{N}$ denotes the $N \times N$ identity matrix. $\mathbf{x} \in \mathcal{C N}(\mathbf{0}, \mathbf{I})$ means $\mathbf{x}$ is a circular symmetric complex Gaussian random vectors whose mean vector is $\mathbf{0}$ and covariance matrix is $\mathbf{I}$. $f(y) \sim o(y)$ means that $\lim _{y \rightarrow \infty} \frac{f(y)}{y}=0$.

\section{SySTEM MOdEL}

We consider the downlink transmission of the multicell multicast network comprising $N$ cells and $K$ mobile users per cell as shown in Fig. 1. The BS in each cell is equipped with $M$ antennas, where $M$ is a large number under massive MIMO, and every mobile user has a single antenna. Let $\mathbf{g}_{i, j, k}^{H}$ denote the flat-fading channel from the BS in the $i$ th cell to the $k$ th user in the $j$ th cell, which can be written as follows

$$
\mathbf{g}_{i, j, k}^{H}=\sqrt{\beta_{i, j, k}} \mathbf{h}_{i, j, k}^{H},
$$

where $\left\{\beta_{i, j, k} \in \mathbb{R}\right\}$ are the large-scale channel attenuation which change slowly and can be obtained rather easily, and $\left\{\mathbf{h}_{i, j, k} \in \mathcal{C N}\left(\mathbf{0}, \mathbf{I}_{M}\right)\right\}$ are the small-scale fading coefficients and modeled as independent and identically distributed (i.i.d.) random vectors. Let $\mathbf{w}_{i}$ denote the $M \times 1$ unit-norm multicasting beamforming vector applied at BS $i$ and $s_{i}$ denote the multicast information symbol for the users in the $i$ th cell. We assume that all the BSs and users are perfectly synchronized, and work in the same time-frequency resource. The discretetime baseband signal received by the $k$ th user in the $i$ th cell is given by

$$
y_{i, k}=\sqrt{p_{i}} \mathbf{g}_{i, i, k}^{H} \mathbf{w}_{i} s_{i}+\sum_{j \neq i}^{N} \sqrt{p_{j}} \mathbf{g}_{j, i, k}^{H} \mathbf{w}_{j} s_{j}+z_{i, k}
$$

where $p_{i}$ is the transmit power by BS $i$, and $z_{i, k}$ is the additive white circularly symmetric Gaussian complex noise with mean zero and variance $\sigma^{2}$. In (2), the second term is the interference from the BSs in other cells. Based on the received signal model in (2), the performance of each user can be characterized by the output SINR, defined as

$$
\operatorname{SINR}_{i, k}=\frac{p_{i}\left|\mathbf{g}_{i, i, k}^{H} \mathbf{w}_{i}\right|^{2}}{\sum_{j \neq i}^{N} p_{j}\left|\mathbf{g}_{j, i, k}^{H} \mathbf{w}_{j}\right|^{2}+\sigma^{2}} .
$$

Here, we have assumed that the signal and interference terms are uncorrelated. For the multicell multicast system, each BS transmits a common message to the $K$ served users. Thus, the performance of each cell is determined by the user with the minimum SINR.

Before moving on to the next section, we review two basic results on random vectors [9] that will be useful later on. Let $\mathbf{x}=\left[x_{1}, \ldots, x_{n}\right]^{T}$ and $\mathbf{y}=\left[y_{1}, \ldots, y_{n}\right]^{T}$ be mutually independent $n \times 1$ vectors whose elements are i.i.d zero-mean random variables with variance being $\sigma_{x}^{2}$ and $\sigma_{y}^{2}$, respectively. Then from the law of large numbers, we have

$$
\lim _{n \rightarrow \infty} \frac{\mathbf{x}^{H} \mathbf{x}}{n} \stackrel{\text { a.s. }}{\rightarrow} \sigma_{x}^{2}, \text { and } \lim _{n \rightarrow \infty} \frac{\mathbf{x}^{H} \mathbf{y}}{n} \stackrel{\text { a.s. }}{\rightarrow} 0
$$

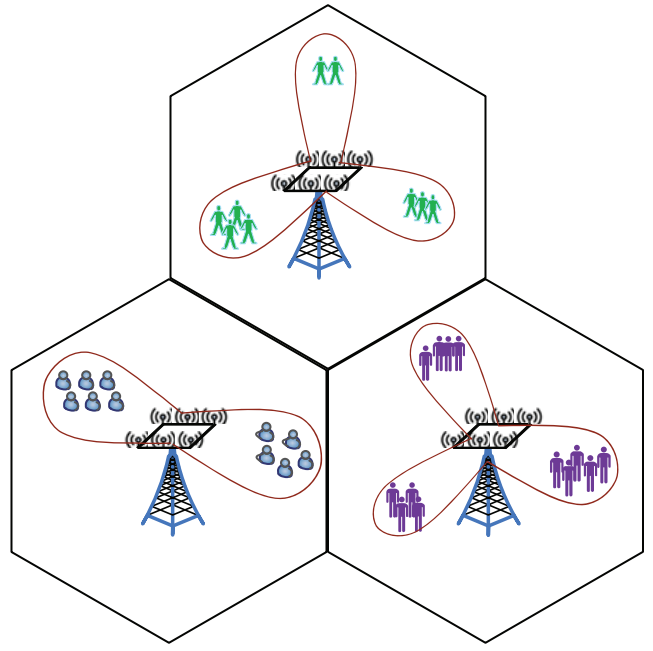

Fig. 1. Multicell multicast network with massive MIMO.

where $\stackrel{\text { a.s. }}{\rightarrow}$ denotes the almost sure convergence.

\section{Asymptotic Analysis with Perfect CSI}

In this section, we consider the asymptotic analysis for the multicell multicast network with massive MIMO when perfect CSI is available. Due to the feature of the multicast transmission, the performance of the worst user is the bottleneck of the whole cell. Thus, for each BS, our goal of the beamforming design is to maximize the minimum SINR of the $K$ served users. This is formulated as the following optimization problem

$$
\begin{gathered}
\mathcal{P}: \max _{\mathbf{w}_{i}} \min _{\forall k} \frac{p_{i}\left|\mathbf{g}_{i, i, k}^{H} \mathbf{w}_{i}\right|^{2}}{\sum_{j \neq i}^{N} p_{j}\left|\mathbf{g}_{j, i, k}^{H} \mathbf{w}_{j}\right|^{2}+\sigma^{2}} \\
\text { s.t. } \quad\left\|\mathbf{w}_{i}\right\|=1 .
\end{gathered}
$$

\section{A. Asymptotically Optimal Beamformer Structure}

The optimization problem $\mathcal{P}$ for each $\mathrm{BS}$ is equivalent to the conventional single-cell multicast beamforming problem in [1]. Due to the NP-hardness, its optimal solution cannot be obtained efficiently in general. In our case, since each BS has a large number of antennas, the different channels become asymptotically orthogonal [10]. Therefore, we are able to derive the asymptotically optimal structure of the beamforming vector in the following theorem.

Theorem 1 : The asymptotically optimal beamformer for each BS when $M \rightarrow \infty$ is a linear combination of the channels between the BS and its served users, i.e.,

$$
\mathbf{w}_{i}=\sum_{k=1}^{K} \xi_{i, k} \mathbf{g}_{i, i, k}, \forall i \in\{1, \ldots, N\}
$$

where $\left\{\xi_{i, k}\right\}$ are the linear combination coefficients.

Proof: The proof is based on the asymptotic orthogonality of the channel vectors when $M$ is large. We first show that with the beamformer of each BS being $\mathbf{w}_{i}=\sum_{k=1}^{K} \xi_{i, k} \mathbf{g}_{i, i, k}$, the 
intercell interference each user suffers vanishes as $M \rightarrow \infty$ :

$$
\begin{aligned}
y_{i, k}= & \sqrt{p_{i}} \mathbf{g}_{i, i, k}^{H} \mathbf{w}_{i} s_{i}+\sum_{j \neq i}^{N} \sqrt{p_{j}} \mathbf{g}_{j, i, k}^{H} \mathbf{w}_{j} s_{j}+z_{i, k} \\
= & \sqrt{p_{i}} \mathbf{g}_{i, i, k}^{H}\left(\sum_{k^{\prime}=1}^{K} \xi_{i, k^{\prime}} \mathbf{g}_{i, i, k^{\prime}}\right) s_{i} \\
& +\sum_{j \neq i}^{N} \sqrt{p_{j}} \mathbf{g}_{j, i, k}^{H}\left(\sum_{k^{\prime}=1}^{K} \xi_{j, k^{\prime}} \mathbf{g}_{j, j, k^{\prime}}\right) s_{j}+z_{i, k} \\
= & \underbrace{\sqrt{p_{i}} \xi_{i, k} \mathbf{g}_{i, i, k}^{H} \mathbf{g}_{i, i, k} s_{i}}_{\Gamma}+o(\Gamma)+z_{i, k} .
\end{aligned}
$$

which can be thought as that the BS applies matched filter beamforming vector, and does not cause interference to the users in the other cells at the same time

If for one of the BSs, say BS $i$, its beamformer does not have this structure, we can write it as follows

$$
\mathbf{w}_{i}^{\prime}=\sum_{k=1}^{K} \xi_{i, k}^{\prime} \mathbf{g}_{i, i, k}+\sum_{t=1}^{M-K} \zeta_{i, t} \mathbf{u}_{i, t}
$$

where $\left\{\mathbf{u}_{i, t}\right\}_{t=1}^{M-K}$ is an orthogonormal basis for the orthogonal complement of the space spanned by $\left\{\mathbf{g}_{i, i, k}\right\}_{k=1}^{K}$.

From (8) it is seen that the component $\sum_{t=1}^{M-K} \zeta_{i, t} \mathbf{u}_{i, t}$ makes no contribution to the useful signals of the users in the $i$ th cell but will cause extra interference to the users in the other cells. Hence, we can always reconstruct another beamformer for this BS without changing the transmit power as

$$
\mathbf{w}_{i}^{\prime \prime}=\sum_{k=1}^{K} \eta \xi_{i, k}^{\prime} \mathbf{g}_{i, i, k}
$$

where

$\eta=\sqrt{\left(\sum_{k=1}^{K}\left|\xi_{i, k}^{\prime}\right|^{2}+\sum_{t=1}^{M-K}\left|\zeta_{i, t}\right|^{2}\right) /\left(\sum_{k^{\prime}=1}^{K}\left|\xi_{i, k}^{\prime}\right|^{2}\right)}>1$.

Give that the beamformers of other cells keep unchanged, the received powers of the users in the $i$ th cell under $\mathbf{w}_{i}^{\prime \prime}$ is larger than that under $\mathbf{w}_{i}^{\prime}$, and the interference caused by $\mathbf{w}_{i}^{\prime \prime}$ to the users in other cells still vanishes. This completes the proof of this theorem.

From Theorem 1, we can see that the massive MIMO beamformer for the multicell multicast network has a rather simple structure. In the rest of the paper, we will apply this structure for the beamforming design.

Then, we will derive the limiting SINR achieved at each user for the considered network. We first normalize the beamforming vector for each $\mathrm{BS} i$ as follows

$$
\mathbf{w}_{i}=\frac{\sum_{k=1}^{K} \xi_{i, k} \mathbf{g}_{i, i, k}}{\alpha_{i} \sqrt{M}}
$$

where the normalization factor is

$$
\alpha_{i}=\frac{\left\|\sum_{k=1}^{K} \xi_{i, k} \mathbf{g}_{i, i, k}\right\|}{\sqrt{M}} .
$$

According to (4), we derive the asymptotic behavior of $\alpha_{i}^{2}$ as below

$$
\begin{aligned}
& \lim _{M \rightarrow \infty} \alpha_{i}^{2}=\lim _{M \rightarrow \infty} \frac{\left(\sum_{k=1}^{K} \xi_{i, k} \mathbf{g}_{i, i, k}\right)^{H}\left(\sum_{k=1}^{K} \xi_{i, k} \mathbf{g}_{i, i, k}\right)}{M} \\
& =\lim _{M \rightarrow \infty} \frac{\sum_{k=1}^{K} \sum_{k^{\prime}=1}^{K}\left|\xi_{i, k}^{*} \xi_{i, k^{\prime}}\right| \sqrt{\beta_{i, i, k} \beta_{i, i, k^{\prime}}} \mathbf{h}_{i, i, k}^{H} \mathbf{h}_{i, i, k^{\prime}}}{M} \\
& =\sum_{k=1}^{K}\left|\xi_{i, k}\right|^{2} \beta_{i, i, k} .
\end{aligned}
$$

Assume the transmit power of each BS $p_{i}$ is scaled with $M$ according to $p_{i}=\frac{E_{i}}{M}$ where $E_{i}$ is fixed. Then, the asymptotic SINR of user $k$, for $k=1, . ., K$ at cell $i$ is derived as below

$$
\begin{aligned}
& \lim _{M \rightarrow \infty} \operatorname{SINR}_{i, k}=\lim _{M \rightarrow \infty} \frac{p_{i}\left|\mathbf{g}_{i, i, k}^{H} \mathbf{w}_{i}\right|^{2}}{\sum_{j \neq i}^{N} p_{j}\left|\mathbf{g}_{j, i, k}^{H} \mathbf{w}_{j}\right|^{2}+\sigma^{2}} \\
& =\lim _{M \rightarrow \infty} \frac{\frac{E_{i} \beta_{i, i, k}}{\alpha_{i}^{2}}\left|\frac{\sum_{k^{\prime}=1}^{K} \xi_{i, k^{\prime}} \sqrt{\beta_{i, i, k^{\prime}}} \mathbf{h}_{i, i, k}^{H} \mathbf{h}_{i, i, k^{\prime}}}{M}\right|^{2}}{\sum_{j \neq i}^{N} \frac{E_{j} \beta_{j, i, k}}{\alpha_{j}^{2}}\left|\frac{\sum_{k^{\prime}=1}^{K} \xi_{j, k^{\prime}} \sqrt{\beta_{j, j, k^{\prime}}} \mathbf{h}_{j, i, k}^{H} \mathbf{h}_{j, j, k^{\prime}}}{M}\right|^{2}+\sigma^{2}} .
\end{aligned}
$$

Applying (4) and plugging (12) into (13), we obtain

$$
\begin{aligned}
\lim _{M \rightarrow \infty} \operatorname{SINR}_{i, k} & =\frac{\left[\frac{\left|\xi_{i, k}\right|^{2} \beta_{i, i, k}}{\sum_{k^{\prime}=1}^{K}\left|\xi_{i, k^{\prime}}\right|^{2} \beta_{i, i, k^{\prime}}}\right] \beta_{i, i, k} E_{i}}{\sigma^{2}} \\
& =\frac{\lambda_{i, k} E_{i} \beta_{i, i, k}}{\sigma^{2}}
\end{aligned}
$$

where

$$
\lambda_{i, k}=\frac{\left|\xi_{i, k}\right|^{2} \beta_{i, i, k}}{\sum_{k^{\prime}=1}^{K}\left|\xi_{i, k^{\prime}}\right|^{2} \beta_{i, i, k^{\prime}}} .
$$

From (14) it is seen that with a large $M$, each BS just needs the power $\frac{E_{i}}{M}$ to achieve the same performance as that of a single-input single-output (SISO) system with transmit power $\lambda_{i, k} E_{i}$, without any interference and the fast-fading effect, which coincides with [11]. For each antenna, its transmit power will be $\frac{E_{i}}{M^{2}}$. Thus, given the SINR target, we can reduce the power consumption at the $\mathrm{BS}$ by increasing the BS antennas. In the rest of this paper, we will assume that $p_{i}=\frac{E_{i}}{M}, \forall i$. 


\section{B. Optimal Beamforming Parameters}

In this subsection, we will derive the optimal linear combination coefficients in the asymptotic beamformer in Theorem 1 for the max-min problem in (5). Recall that the limiting SINR is directly related to $\left\{\lambda_{i, k}\right\}$ instead of $\left\{\xi_{i, k}\right\}$ as defined in (15). Moreover, an important observation is that

$$
\sum_{k=1}^{K} \lambda_{i, k}=1 .
$$

Therefore, optimizing the coefficients $\left\{\xi_{i, k}\right\}$ is equivalent to finding the optimal $\left\{\lambda_{i, k}\right\}$. This problem can be formulated as

$$
\mathcal{Q}: \max _{\left\{\lambda_{i, k}\right\}} \min _{\forall k} \frac{\lambda_{i, k} E_{i} \beta_{i, i, k}}{\sigma^{2}}
$$

Lemma $2^{1}$ : The optimal solution of problem $\mathcal{Q}$ is obtained when all the $K$ users have the same SINR, i.e.,

$$
\frac{\lambda_{i, 1} E_{i} \beta_{i, i, 1}}{\sigma^{2}}=\ldots=\frac{\lambda_{i, K} E_{i} \beta_{i, i, K}}{\sigma^{2}}
$$

and the resulting $\left\{\lambda_{i, k}\right\}$ are

$$
\lambda_{i, k}=\frac{1}{\sum_{k^{\prime}=1}^{K} \frac{\beta_{i, i, k}}{\beta_{i, i, k^{\prime}}}}, \forall i, \forall k .
$$

According to Lemma 2, we can obtain the asymptotically optimal normalized beamformer as follows

$$
\mathbf{w}_{i}=\mu_{i} \sum_{k=1}^{K} \frac{\mathbf{g}_{i, i, k}}{\beta_{i, i, k}}
$$

where the common factor $\mu_{i}$ is

$$
\mu_{i}=\frac{1}{\sqrt{M \sum_{k=1}^{K} \frac{1}{\beta_{i, i, k}}}} .
$$

We can see that the user with large channel gain (i.e large $\beta$ ) will have less weight in the beamformer.

\section{A Contamination-Free Pilot Scheme}

In this section, we consider the imperfect CSI scenario and each BS obtains the CSI by doing channel estimation in the uplink. In order to avoid pilot contamination, a simple and straightforward way is to assign a different and orthogonal pilot sequence to each user in the whole network. However, this will need a very long pilot sequence which becomes prohibitive for practical scenarios. In this paper, we propose a novel pilot design for the multicell multicast network with large scale antenna arrays to eliminate the pilot contamination. The key idea is that we apply a set of $N$ orthogonal pilot sequences for the whole network and the $K$ users in each cell share the same pilot sequence. Thus, each BS will obtain an estimated composite channel of the served $K$ users.

\footnotetext{
${ }^{1}$ The proof of this lemma is omitted for space limitation.
}

For cell $i$, each of the $K$ users uses the same $1 \times \omega$ pilot sequence

$$
\sqrt{\omega} \psi_{i}=\sqrt{\omega}\left[\psi_{i 1}, \ldots, \psi_{i \omega}\right]
$$

where $\omega \geq N$ and $\boldsymbol{\psi}_{i} \boldsymbol{\psi}_{j}^{H}=\delta_{i, j}$. BS $i$ then receives the following signal during the pilot transmission phase

$$
\mathbf{Y}_{B_{i}}=\sum_{l=1}^{N} \sum_{k=1}^{K} \sqrt{p_{u} \omega} \mathbf{g}_{i, l, k} \boldsymbol{\psi}_{l}+\mathbf{Z}_{B_{i}}
$$

BS $i$ then estimates the composite channel vector for the users in cell $i$ as

$$
\begin{aligned}
\hat{\mathbf{g}}_{i, c} & =\mathbf{Y}_{B_{i}} \boldsymbol{\psi}_{i}^{H} \\
& =\sum_{l=1}^{N} \sum_{k=1}^{K} \sqrt{p_{u} \omega} \mathbf{g}_{i, l, k} \boldsymbol{\psi}_{l} \boldsymbol{\psi}_{i}^{H}+\mathbf{Z}_{B_{i}} \boldsymbol{\psi}_{i}^{H} \\
& =\sum_{k=1}^{K} \sqrt{p_{u} \omega} \mathbf{g}_{i, i, k}+\mathbf{z}_{B_{i}}
\end{aligned}
$$

Upon obtaining the composite CSI, the BS can design the beamforming vector as

$$
\begin{aligned}
\mathbf{w}_{i} & =\frac{\hat{\mathbf{g}}_{i, c}}{\left\|\hat{\mathbf{g}}_{i, c}\right\|} \\
& =\sum_{k=1}^{K} \frac{\sqrt{p_{u} \omega}}{\left\|\hat{\mathbf{g}}_{i, c}\right\|} \mathbf{g}_{i, i, k}+\frac{\mathbf{z}_{B_{i}}}{\left\|\hat{\mathbf{g}}_{i, c}\right\|} .
\end{aligned}
$$

Thus, there will be no intercell interference for the proposed pilot scheme. Also this beamformer has the asymptotically optimal structure derived in Theorem 1 since it is the linear combination for the channel vectors of the served $K$ users, except the additive noise which cannot be avoided. However, the BS itself is not able to optimize these coefficients for the beamformer since it does not know each user's individual channel.

On the other hand, from (23) we can see that the pilot transmit power of each user is contained in the composite CSI obtained by the BS. This indicates that we can change each user's pilot transmit power to change the composite channel estimated by the BS. Thus, the other key point is that we can do power control among the $K$ users of each cell in the pilot transmission phase to optimize the coefficients in each BS's beamformer, and to improve the network performance.

Denoting the transmit power of user $k$ in the pilot transmission phase as $p_{i, k}$, we can rewrite (23) as below

$$
\hat{\mathbf{g}}_{i, c}=\sum_{k=1}^{K} \sqrt{\omega \beta_{i, i, k} p_{i, k}} \mathbf{h}_{i, i, k}+\mathbf{z}_{B_{i}} .
$$

Then the normalized beamforming vector is

$$
\mathbf{w}_{i}=\frac{\sum_{k=1}^{K} \sqrt{\omega \beta_{i, i, k} p_{i, k}} \mathbf{h}_{i, i, k}+\mathbf{z}_{B_{i}}}{\mu_{i} \sqrt{M}}
$$


where $\mu_{i}=\frac{\left\|\sum_{k=1}^{K} \sqrt{\omega \beta_{i, i, k} p_{i, k}} \mathbf{h}_{i, i, k}+\mathbf{z}_{B_{i}}\right\|}{\sqrt{M}}$ and its limiting value is given by

$$
\lim _{M \rightarrow \infty} \mu_{i}^{2}=\omega \sum_{k=1}^{K} \beta_{i, i, k} p_{i, k}+\sigma^{2} .
$$

With the above beamformer, we derive the limiting SINR of each user when $M \rightarrow \infty$ for the proposed pilot scheme as follows

$$
\begin{aligned}
& \lim _{M \rightarrow \infty} \operatorname{SINR}_{i, k} \\
&= \lim _{i} \beta_{i, i, k} \frac{\left.\mathbf{h}_{i, i, k}^{H} \frac{\left(\sum_{k^{\prime}=1}^{K} \sqrt{\omega \beta_{i, i, k^{\prime}} p_{i, k^{\prime}}} \mathbf{h}_{i, i, k^{\prime}}+\mathbf{z}_{B_{i}}\right)}{\mu_{i} \sqrt{M}}\right|^{2}}{\sum_{j \neq i}^{N} p_{j} \beta_{j, i, k}\left|\mathbf{h}_{j, i, k}^{H} \frac{\left(\sum_{k^{\prime}=1}^{K} \sqrt{\omega \beta_{j, j, k^{\prime}} p_{j, k^{\prime}}} \mathbf{h}_{j, j, k^{\prime}}+\mathbf{z}_{B_{i}}\right)}{\mu_{i} \sqrt{M}}\right|+\sigma^{2}} \mid \\
& \mid
\end{aligned}
$$$$
=\frac{E_{i} \beta_{i, i, k}^{2} \omega p_{i, k}}{\mu_{i}^{2} \sigma^{2}}
$$$$
=\frac{E_{i}}{\sigma^{2}} \cdot \frac{\beta_{i, i, k}^{2} p_{i, k}}{\sum_{k^{\prime}=1}^{K} \beta_{i, i, k^{\prime}} p_{i, k^{\prime}}+\frac{\sigma^{2}}{\omega}} .
$$

Based on the max-min criterion, we can formulate the pilot power control problem for each cell as follows

$$
\begin{aligned}
\mathcal{T}: \max _{\left\{p_{i, k}\right\}} \min _{\forall k} \frac{\beta_{i, i, k}^{2} p_{i, k}}{\sum_{k^{\prime}=1}^{K} \beta_{i, i, k^{\prime}} p_{i, k^{\prime}}+\frac{\sigma^{2}}{\omega}} \\
\text { s.t. } \quad p_{i, k} \leq p_{u}, \forall k \in\{1, \ldots, K\} .
\end{aligned}
$$

where $p_{u}$ denotes the peak transmit power of each user.

Changing the objective function of $\mathcal{T}$, we can rewrite the problem as

$$
\begin{aligned}
\mathcal{T}: \min _{\left\{p_{i, k}\right\}} & \max _{\forall k} \frac{\sigma^{2}}{\omega} \beta_{i, i, k}^{-2} p_{i, k}^{-1}+\sum_{k^{\prime}=1}^{K} \beta_{i, i, k}^{-2} \beta_{i, i, k^{\prime}} p_{i, k}^{-1} p_{i, k^{\prime}} \\
& \text { s.t. } \quad p_{i, k} \leq p_{u}, \forall k \in\{1, \ldots, K\} .
\end{aligned}
$$

To further simplify the objective function of the above optimization problem, we introduce a slack variable $t$ and reformulate the problem as follows

$$
\begin{array}{ll}
\mathcal{T}: & \min _{\left\{p_{i, k}, t\right\}} t \\
\text { s.t. } & \frac{\sigma^{2}}{\omega} \beta_{i, i, k}^{-2} p_{i, k}^{-1} t^{-1}+\sum_{k^{\prime}=1}^{K} \beta_{i, i, k}^{-2} \beta_{i, i, k^{\prime}} p_{i, k}^{-1} p_{i, k^{\prime}} t^{-1} \leq 1 \\
& p_{i, k} \leq p_{u}, \forall k \in\{1, \ldots, K\} .
\end{array}
$$

The above problem is a geometric programming problem. It can be transformed to be a convex problem and solved optimally using the software package [12].

Remark 1: In the proposed pilot scheme for composite channel estimation, the length of the pilot should not be less than $N$; Recall that if the individual channel estimation scheme is applied, the pilot length will be at least $K$. Similar to user number $K$, the cell number $N$ (adjacent cells) will not be too large and be proper for practical implementations.

Remark 2: In the optimal pilot power control, the knowledge of large-scale channel attenuation factors of all users in the same cell should be known. In practice, the channel attenuation changes very slowly compared with the small-scale fading, and hence can be estimated in advance using off-line approaches.

\section{NumericAl RESUlts}

In this section, we provide numerical results to illustrate the asymptotic performance of the considered multicell multicast network with massive MIMO. The cells of the network are hexagonal with a radius (from center to vertex) of $r=1000$ meters. The mobile users are randomly distributed in each cell with uniform distribution, excluding an inner circle of $r_{0}=100$ meters. The channel bandwidth is $20 \mathrm{MHz}$. For the large-scale fading, the distance-dependent path loss in $\mathrm{dB}$ is modeled as $P L_{N L O S}=128.1+37.6 \log _{10}(d)$, where $d$ is the distance from the user to the BS in kilometers. The lognormal shadowing is considered with $\sigma_{\text {shadow }}=8 \mathrm{~dB}$ and the penetration loss is assumed to be $20 \mathrm{~dB}$. The small-scale fading is assumed as the normalized Rayleigh fading. The noise power spectral density is set to be $-174 \mathrm{dBm} / \mathrm{Hz}$, and we set $\sigma_{p}^{2}=0.1 \sigma^{2}$. Throughout this section, we consider the network containing 7 cells and choose the cell in the center for performance evaluation, which also represents the worst scenario. For each analytical result, 1000 independent largescale channel realizations are generated.

We first demonstrate the effectiveness of the asymptotically optimal beamformer in the perfect CSI case. In Fig. 2, we plot the CDF (Cumulative Distribution Function) curve of the minimum asymptotic SINR at all $K$ users in the central cell. We compute the SINR according to (14). For the 'optimal parameter' case, the parameters $\left\{\lambda_{i, k}\right\}$ are set as in (19); For the 'equal parameter' case, the parameters $\left\{\lambda_{i, k}\right\}$ are set as in (15) with $\left\{\xi_{i, k}\right\}$ being 1 . We can see that at $50 \%$ SINR our proposed algorithm outperforms the simple equal parameter design by $10 \mathrm{~dB}$ for $K=3$. We can also see that the achieved SINR decreases when the user number increases in the multicast network.

For the imperfect CSI scenario, the length of the pilot sequence is set to be 8 . The pilot peak transmit power is assumed as $p_{u}=2 \mathrm{~dB} W$. In Fig. 3, we investigate the performance of the proposed pilot scheme. The asymptotic SINR of the pilot scheme for composite channel estimation is computed according to (28). For the 'composite \& optimized' case, the pilot power is obtained by solving problem (29); For the 'composite \& unoptimized' case, the pilot power of each user is set to be $p_{u}$. It can be seen that the performance of the proposed pilot scheme with optimal power control is close to the perfect CSI case with optimal parameter design. When applying pilot power control, we can obtain significant performance gain. Specifically, the achieved gain at $50 \%$ SINR is $5 \mathrm{~dB}$ in the network with 3 users in each cell. 


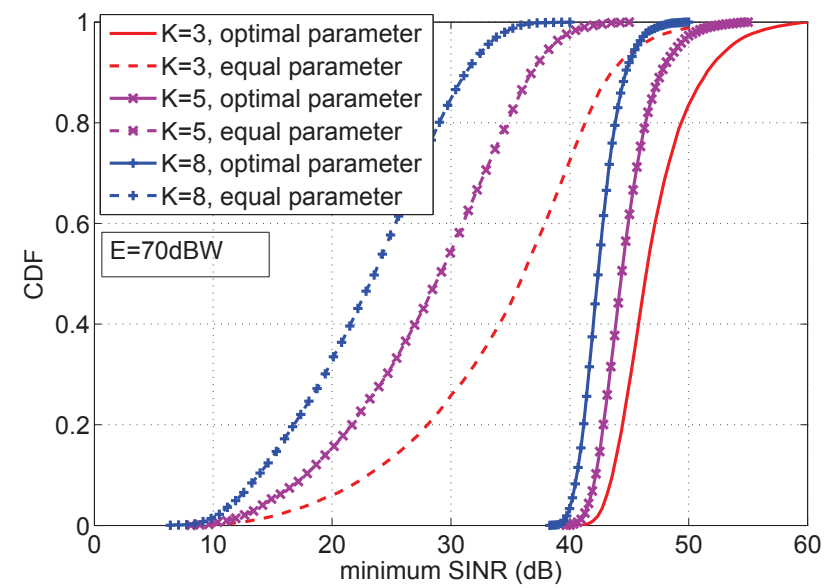

Fig. 2. Comparison on the asymptotic achieved SINR of the proposed optimal parameter design with equal parameter design under the perfect CSI scenario.

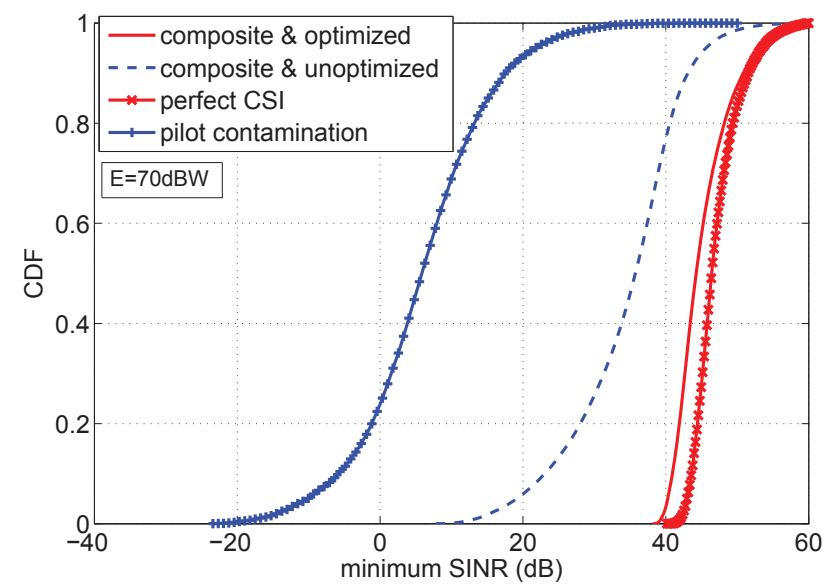

Fig. 3. The CDF curves of the achieved asymptotic SINR for the proposed pilot scheme and the perfect CSI case when each cell has $K=3$ users.

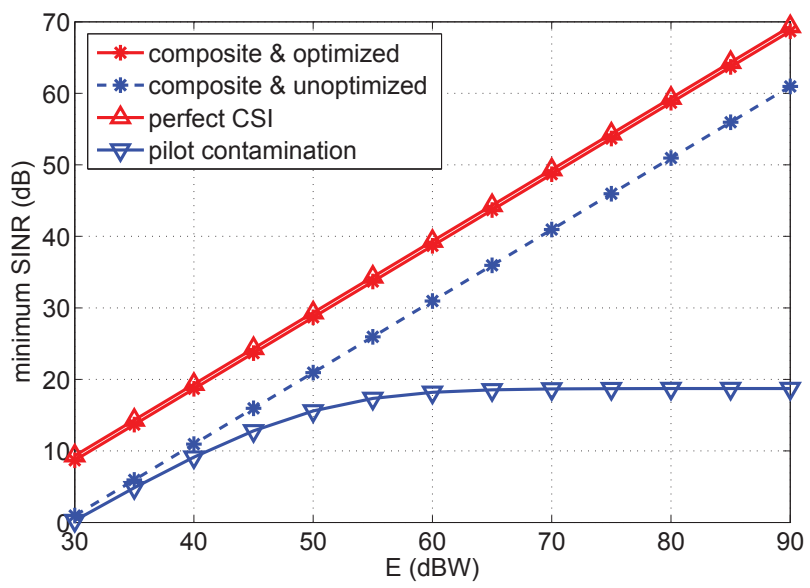

Fig. 4. Comparison on the performance of the proposed pilot scheme for composite channel estimation with the perfect CSI case when each cell has $K=3$ users.
In Fig. 4, we plot the asymptotic SINR curves for different $E$ 's and similar phenomenon can be seen. We also observe that when increasing $E$, the mininum SINR increases almost linearly. This is in contract to the conventional channel estimation case where SINR ceiling would occur due to pilot contamination.

\section{CONCLUSION}

This paper considered the noncooperative multicell multicast network with massive MIMO. For the perfect CSI case, we derived the asymptotically optimal beamformer structure for each BS. The optimal parameter in the beamforming vector with closed-form expression was also derived. For the imperfect CSI, we proposed a contamination-free pilot scheme for composite channel estimation. Also, by doing power control among users in the pilot transmission stage, our proposed scheme has close performance to the perfect CSI scenario.

\section{REFERENCES}

[1] N. D. Sidiropoulos, T. N. Davidson, and Z.-Q. Luo, "Transmit beamforming for physical-layer multicasting," IEEE Transactions on Signal Processing, vol. 54, no. 6, pp. 2239-2251, Jun. 2006.

[2] E. Karipidis, N. D. Sidiropoulos, and Z.-Q. Luo, "Quality of service and max-min fair transmit beamforming to multiple cochannel multicast groups," IEEE Transactions on Signal Processing, vol. 56, no. 3, pp. 1268-1279, 2008.

[3] Z. Xiang, M. Tao, and X. Wang, "Coordinated multicast beamforming in multicell networks," IEEE Transactions on Wireless Communications, vol. 12, no. 1, pp. 12-21, Jan. 2013.

[4] S. X. Wu, A. M.-C. So, and W.-K. Ma, "Rank-two transmit beamformed alamouti space-time coding for physical-layer multicasting," in IEEE International Conference on Acoustics, Speech and Signal Processing (ICASSP), 2012, pp. 2793-2796.

[5] X. Wen, K. L. Law, S. J. Alabed, and M. Pesavento, "Rank-two beamforming for single-group multicasting networks using OSTBC," in IEEE 7th Sensor Array and Multichannel Signal Processing Workshop (SAM), 2012, pp. 69-72.

[6] S. X. Wu and W.-K. Ma, "Multicast transmit beamforming using a randomize-in-time strategy," in IEEE International Conference on Acoustics, Speech and Signal Processing (ICASSP), 2011, pp. 33763379.

[7] F. Rusek, D. Persson, B. K. Lau, E. Larsson, T. Marzetta, O. Edfors, and F. Tufvesson, "Scaling up MIMO: Opportunities and challenges with very large arrays," IEEE Signal Processing Magazine, vol. 30, no. 1, pp. 40-60, Jan. 2013

[8] T. L. Marzetta, "Noncooperative cellular wireless with unlimited numbers of base station antennas," IEEE Transactions on Wireless Communications, vol. 9, no. 11, pp. 3590-3600, Nov. 2010.

[9] H. Cramér, Random variables and probability distributions. Cambridge University Press, 2004, no. 36.

[10] E. G. Larsson, F. Tufvesson, O. Edfors, and T. L. Marzetta, "Massive mimo for next generation wireless systems," arXiv:1304.6690, 2013.

[11] H. Q. Ngo, E. Larsson, and T. Marzetta, "Energy and spectral efficiency of very large multiuser mimo systems," IEEE Transactions on Communications, vol. 61, no. 4, pp. 1436-1449, Apr. 2013.

[12] M. Grant and S. Boyd, CVX: Matlab software for disciplined convex programming. Available: http://stanford.edu.boyd/cvx. 